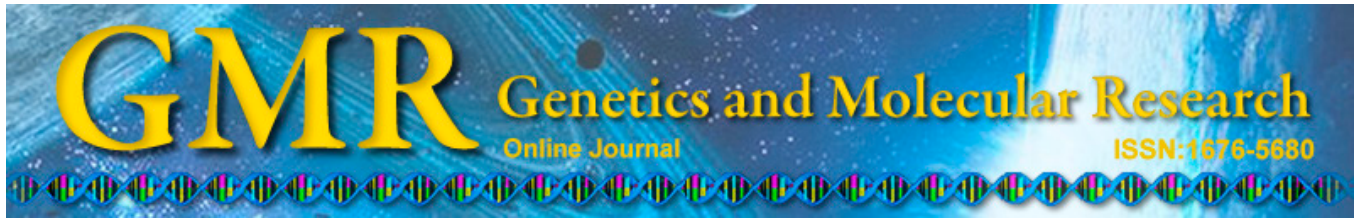

\title{
Forkhead box protein 01 mediates apoptosis in a cancer cervical cell line treated with the antitumor agent tumor necrosis factor- $\alpha$
}

\author{
B. Zhang ${ }^{1,2}$, L. Gui ${ }^{1}$, L. Zhu ${ }^{2}$, X. Zhao ${ }^{2}$, Y. Yang ${ }^{2}$ and Q. Li ${ }^{1}$ \\ ${ }^{1}$ College of Animal Science and Technology, Northwest A \& F University, \\ Yangling, Shaanxi, China \\ ${ }^{2}$ College of Basic Medical Sciences, Jiamusi University, Jiamusi, \\ Heilongjiang, China \\ Corresponding author: Q. Li \\ E-mail: liqingwangysu@aliyun.com
}

Genet. Mol. Res. 14 (3): 7446-7454 (2015)

Received September 9, 2014

Accepted December 11, 2014

Published July 3, 2015

DOI http://dx.doi.org/10.4238/2015.July.3.20

\begin{abstract}
Tumor necrosis factor- $\alpha$ (TNF- $\alpha)$ is an important proapoptotic cytokine, which performs a broad range of immune and inflammatory functions in several vital processes. TNF- $\alpha$-induced apoptosis has been confirmed, however, relatively little is known regarding the role of forkhead box class-O 1 (FOXO1) in mediating TNF- $\alpha$-induced apoptosis in cervical cancer. In our study, we used the well-characterized cervical cancer cell line C-33A to investigate the role of FOXO1. The results showed that the antitumor agent TNF- $\alpha$ increased the expression level of FOXO1 $(\mathrm{P}<0.05)$ and enhanced its transcriptional activity $(\mathrm{P}<0.05)$. Furthermore, knockdown of FOXO1 repressed TNF- $\alpha$-induced apoptosis and caspase- 3,8 , and 9 expressions $(\mathrm{P}<0.05)$. Collectively, these findings suggest that TNF- $\alpha$ upregulated the transcriptional factor FOXO1, leading to an increased expression of apoptotic gene, which leads to an increase in apoptosis.
\end{abstract}

Key words: Cervical cancer; C-33A; TNF- $\alpha$; FOXO1; Apoptosis; Caspase 


\section{INTRODUCTION}

Tumor necrosis factor- $\alpha$ (TNF- $\alpha)$ is an important pro-apoptotic cytokine, which performs a broad range of immune and inflammatory functions in several vital processes. TNF- $\alpha$ exerts its broad range of biological activities by activating several different signaling pathways that can affect diverse functions such as inflammation, survival, or cell death (Navarro and Mora, 2005; Navarro and Mora-Fernandez, 2006). TNF- $\alpha$-induced apoptosis has been confirmed, however, the potential mechanism through which TNF- $\alpha$ induces apoptosis via the activation of transcription factors remains unclear.

A class of candidate transcription factors that could mediate pro-apoptotic genes comprise the forkhead box class-O (FOXO) winged helix transcription factors (Birkenkamp and Coffer, 2003). The mammalian FOXO family consists of four family members: FOXO1 (FKHR), FOXO3 (FKHRL1), FOXO4 (AFX), and FOXO6, which share the highly conserved forkhead DNA-binding domain (Burgering and Kops, 2002; Accili and Arden, 2004; Furukawa-Hibi et al., 2005). The FOXO factors have been shown to play crucial roles in a variety of biological processes, including gluconeogenesis, cell cycle arrest, apoptosis, atrophy, and oxidative and stress response (Calnan and Brunet, 2008).

The transcription factor FOXO1 is essential for regulating cell death, inhibiting cell cycle progression, and modulating differentiation in various cell types. Accumulating evidence considers FOXO1 as a tumor suppressor and inducer of the expression of a large number of pro-apoptotic extracellular and intracellular mediators such as Fas ligand (FasL), TNF-related apoptosis-inducing ligand (TRAIL), and caspases (Brunet et al., 1999; Alikhani et al., 2005a). In numerous cell types, the activation of the FOXO family leads to apoptosis, particularly when its expression or activation is prolonged (Burgering and Medema, 2003; Gilley et al., 2003). Because of the increased expression of pro-apoptotic factors, caspase activity is also increased, and apoptosis is induced (Alikhani et al., 2004; Huang and Tindall, 2007).

In the attempt to better understand the mechanistic process of TNF- $\alpha$-induced apoptosis, the goal of the present study was to explore the transcription factor FOXO1-mediated TNF- $\alpha$-induced apoptosis in a human cervical cancer C-33A cell line.

\section{MATERIAL AND METHODS}

\section{Cell culture and media}

The cervical cancer C-33A cells used in the study were obtained from the Shanghai Institutes for Biological Sciences (Shanghai, China). Cells were cultured in Dulbecco's modified Eagle medium (DMEM, Invitrogen, USA) supplemented with $10 \%$ fetal bovine serum (Hyclone, USA), $100 \mathrm{U} / \mathrm{mL}$ penicillin, and $100 \mathrm{U} / \mathrm{mL}$ streptomycin in a humidified atmosphere containing $5 \% \mathrm{CO}_{2}$ at $37^{\circ} \mathrm{C}$.

Recombinant human TNF- $\alpha$ was purchased from Sigma-Aldrich (USA). TNF- $\alpha$ was dissolved in sterile phosphate buffered saline (PBS) containing $0.1 \%$ endotoxin-free recombinant human serum albumin.

\section{Immunocytochemistry}

Cells were seeded on 24-well plates in $500 \mu 1$ of DMEM and incubated overnight. 
DMEM was removed, and the cells were fixed in 4\% formaldehyde for $15 \mathrm{~min}$ at room temperature. After washing briefly with cold Tris-buffered saline (TBS), $\mathrm{pH} 7.4$, cells were permeabilized with $0.5 \%$ Triton X-100 for 10 min. After blocking with $10 \%$ normal goat serum, the cells were incubated with anti-human FOXO1 (1:50, sc-374427, Santa Cruz, CA, USA) at $4{ }^{\circ} \mathrm{C}$ overnight. This was followed by incubation with a goat anti-mouse IgG-FITC $(1: 100$, Bioss, Beijing, China) for $1 \mathrm{~h}$ at $37^{\circ} \mathrm{C}$. Antibodies were removed from the cells by rinsing with TBS, following which they were mounted on slides with $90 \%$ glycerol. Fluorescence was immediately observed by using an Olympus DP72 microscope (Tokyo, Japan).

\section{Small interfering RNA (siRNA) knockdown experiments}

Experiments were carried out in C-33A cells seeded on 6-well plates that were at 70\% confluence and then transfected with the siRNA or appropriate scramble controls by using the Lipofectamine 2000 transfection reagent following manufacturer instructions. The siRNA duplexes and Lipofectamine 2000 were obtained from Invitrogen (Carlsbad, CA, USA). Three different siRNAs for the human FOXO1 (siRNA-1325, siRNA-1461, and siRNA-1731) were designed based on standard criteria. They were named based on the position of the first base pair in FOXO1 mRNA. Control siRNA duplexes did not have any silencing effect.

\section{Cell apoptosis}

Cell apoptosis was measured by flow cytometry. The harvested cells $\left(1 \times 10^{6}\right)$ were washed with cold PBS and fixed in cold $70 \%$ ethanol overnight. For flow cytometric analysis, the cells were incubated with $40 \mu \mathrm{g} / \mathrm{mL}$ propidium iodide and $100 \mu \mathrm{g} / \mathrm{mL}$ DNase-free RNase $\mathrm{A}$ in PBS at $37^{\circ} \mathrm{C}$ for $30 \mathrm{~min}$. Cells were analyzed by fluorescence-activated cell sorting on an FACSCalibur flow cytometer (San Francisco, CA, USA).

\section{Quantitative reverse transcription-polymerase chain reaction (qRT-PCR)}

Total RNA was extracted from $1 \times 10^{6}$ cells by using Trizol reagent (Invitrogen) according to manufacturer instructions and used for qRT-PCR analysis. Next, $1 \mathrm{~g}$ total RNA was reverse transcribed into complementary DNA by using the Prime Script RT reagent kit (Takara, Shiga, Japan). The qPCRs were performed in an IQ5 detection system (Bio-Rad, Hercules, CA, USA) by using the SYBR Premix Ex TaqTM (TaKaRa). Relative gene expression was determined by using the CT method. Primers used for amplifications were as follows: $\beta$-actin-sense (5'-gacgtggacatccgcaaag-3') and $\beta$-actin-antisense (5'-ctggaaggtggacagcgagg-3'); caspase-8-sense (5'-gggtaatgacaatctcggactct-3') and caspase8 -antisense (5'-ttcaaaggtcgtggtcaaagc-3'); caspase-9-sense (5'-tctggaggatttggtgatgtc-3') and caspase-9-antisense (5'-cattttcttggcagtcaggtc); caspase-3-sense (5'-atcacagcaaaaggagcagttt-3') and caspase-3-antisense (5'-acaccactgtctgtctcaatgc-3'). Beta actin, a non-regulated housekeeping gene was used as an internal control to normalize the input of cDNA. Experiments were performed in triplicate and repeated at least three times.

\section{Western blotting}

Total protein was quantified by using the BCA Reagent Kit (Pierce, Rockford, IL, 
USA), with bovine serum albumin as a standard. Equal amounts of protein $(20 \mu \mathrm{g})$ from each sample were size-fractionated by $8-12 \%$ sodium dodecyl sulfate-polyacrylamide gel electrophoresis (SDS-PAGE) and transferred onto a polyvinylidene fluoride membrane by using a Bio-Rad wet blot transfer apparatus. The membranes were blocked in 5\% skim milk in PBS containing $0.01 \%$ Tween 20 at room temperature for $1 \mathrm{~h}$. The membranes were probed with primary antibodies specific for FOXO1, Caspase-3 (25546-1-AP, San Ying, Wu Han), Caspase-8 (13423-1-AP, San Ying, Wu Han), and Caspase-9 (10380-1-AP, San Ying, Wu Han) at $4{ }^{\circ} \mathrm{C}$ overnight. They were then incubated with goat anti-mouse (bs-0296G-HRP, Bio-Synthesis, Beijing, China) or anti-rabbit secondary antibodies (bs-0295G-HRP, Bio-Synthesis, Beijing, China) for $1 \mathrm{~h}$ and visualized by enhanced chemiluminescence. Western blotting quantification was performed by using the Photoshop software (Berkeley, CA, USA). We evaluated the gene expression as an optical densitometric (OD) ratio, which was scored as the density of the gene fluorescent reactivity relative to that of $\beta$-actin.

\section{Statistical analysis}

All statistical analyses were carried out by using the Statistical Package for the Social Sciences version 17.0 software (SPSS, Inc., Chicago, IL, USA). Analysis was performed with the one-way analysis of variance (ANOVA). Data are reported as means \pm SD for experiments performed at least three times. Results were considered to be statistically significant with $\mathrm{P}$ value $<0.05$.

\section{RESULTS}

\section{TNF- $\alpha$ increases the expression of FOXO1}

The C-33A cells were stimulated with TNF- $\alpha$, and its effect on the expression of FOXO1 was examined by immunocytochemistry (Figure 1A), and mRNA levels were determined by qRT-PCR (Figure 1B and C). In the absence of TNF- $\alpha$, the expression level of FOXO1 was markedly decreased as compared with TNF- $\alpha$ treatment $(\mathrm{P}<0.05)$. Moreover, the TNF- $\alpha$ stimulated the expression of FOXO1 in a dose- (Figure 1B) and time-dependent manner (Figure 1C) as compared to the unstimulated cells $(\mathrm{P}<0.05)$. The optimal TNF- $\alpha$ concentration and time for stimulating FOXO1 expression were $20 \mathrm{ng} / \mathrm{mL}$ and $24 \mathrm{~h}$, respectively. These results showed that TNF- $\alpha$ induced FOXO1 expression and enhanced the transcriptional activity of FOXO1.

\section{Identification of siRNA for the knockdown of FOXO1 expression}

These experiments were carried out to silence FOXO1. Three siRNAs, specific for knocking down human FOXO1 were designed and designated as siRNA-1325, siRNA-1461, and siRNA-1731. The sequence of siRNAs is summarized in Table 1. Cells were seeded on 6-well plates and transfected with different siRNAs for $6 \mathrm{~h}$. After total RNA isolation, the level of FOXO1 expression was examined by qRT-PCR. The mRNA level of FOXO1 was decreased significantly by siRNA-1461 but not siRNA-1461 and $1731(\mathrm{P}<0.05)$. The siRNA-1461 was the most effective and therefore used in further experiments. The effects of each siRNA on silencing FOXO1 are shown in Figure 2. 
a
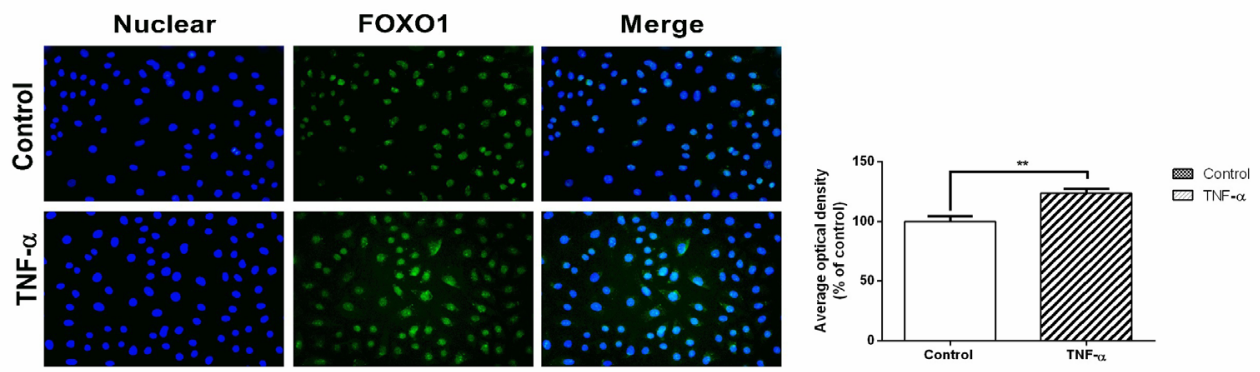

b

C
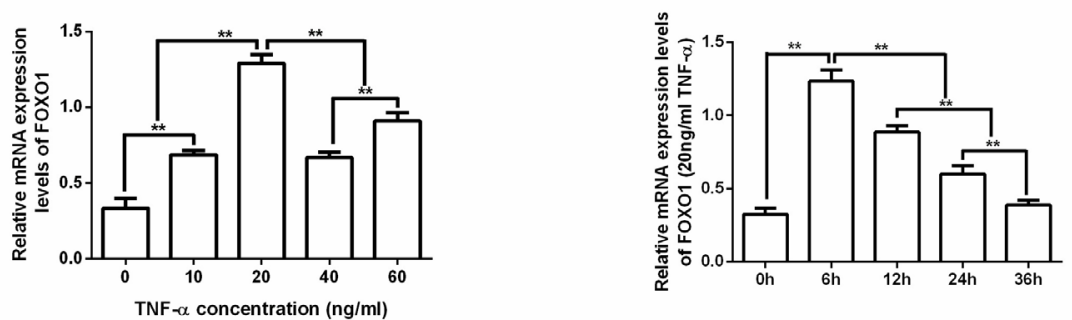

Figure 1. A. Tumor necrosis factor (TNF)- $\alpha$ increases the level of Forkhead box protein O1 (FOXO1) expression. The effect of TNF- $\alpha$ on FOXO1 expression and activation was measured by quantitative reverse transcription polymerase chain reaction. B. C-33A cells were incubated with increasing concentration of TNF- $\alpha$ for $12 \mathrm{~h}$. Total RNA was extracted from cells treated with TNF- $\alpha$ at $0,10,20,40$, or $60 \mathrm{ng} / \mathrm{mL}$. TNF- $\alpha(20 \mathrm{ng} / \mathrm{mL})$ was the optimal concentration $(\mathrm{P}<0.05)$. C. Cells were incubated with $20 \mathrm{ng} / \mathrm{mL}$ TNF- $\alpha$ for increasing length of time. Total RNA was extracted from cells treated with $20 \mathrm{ng} / \mathrm{mL} \mathrm{TNF}-\alpha$ for $0,6,12$, and $24 \mathrm{~h}$. Treatment with TNF- $\alpha$ at the $6-\mathrm{h}$ time point showed the highest expression of FOXO1 $(\mathrm{P}<0.05)$. Results are reported as means \pm SD for three separate experiments. ${ }^{* *} \mathrm{P}<0.05$. The experiments were performed three times with similar results.

Table 1. Sequence of several different siRNA used in silencing FOXO1.

\begin{tabular}{ll}
\hline siRNA & Sequence \\
\hline siRNA-1325 & Sense: 5'CCCUCGAACUAGCUCAAAUTT 3' \\
siRNA-1461 & Antisense: 5'UUUGAGCUAGUUCGAGGGTT3' \\
siRNA-1731 & Sense: 5'CCCAGUCUGUCUGAGAUAATT3' \\
& Antisense: 5'UUAUCUCAGACAGACUGGGTT3' \\
& Sense: 5'GAGGUAUGAGUCAGUAUATT3' \\
\hline
\end{tabular}

\section{Inhibition of FOXO1 blocks TNF- $\alpha$-induced apoptosis}

After FOXO1 had been knocked down, its role in the TNF- $\alpha$-induced apoptosis was investigated by flow cytometry. The C-33A cells were transfected with siRNA-1461 for $6 \mathrm{~h}$ and then treated with $20 \mathrm{ng} / \mathrm{mL}$ TNF- $\alpha$ for $24 \mathrm{~h}$. Control cells were seeded similarly but not treated with TNF- $\alpha$. As expected, siRNA-1461 alone and siRNA-1461 plus TNF- $\alpha$ had very little effect on apoptosis $(\mathrm{P}<0.05)$. However, treatment with TNF- $\alpha$ without siRNA-1461 caused a significant increase in apoptosis as compared with the other groups $(\mathrm{P}<0.05)$. Collectively, 
these results indicate that the silencing of FOXO1 significantly repressed TNF- $\alpha$-induced apoptosis in cervical cancer C-33A cells. The results are shown in Table 2 and Figure 3.

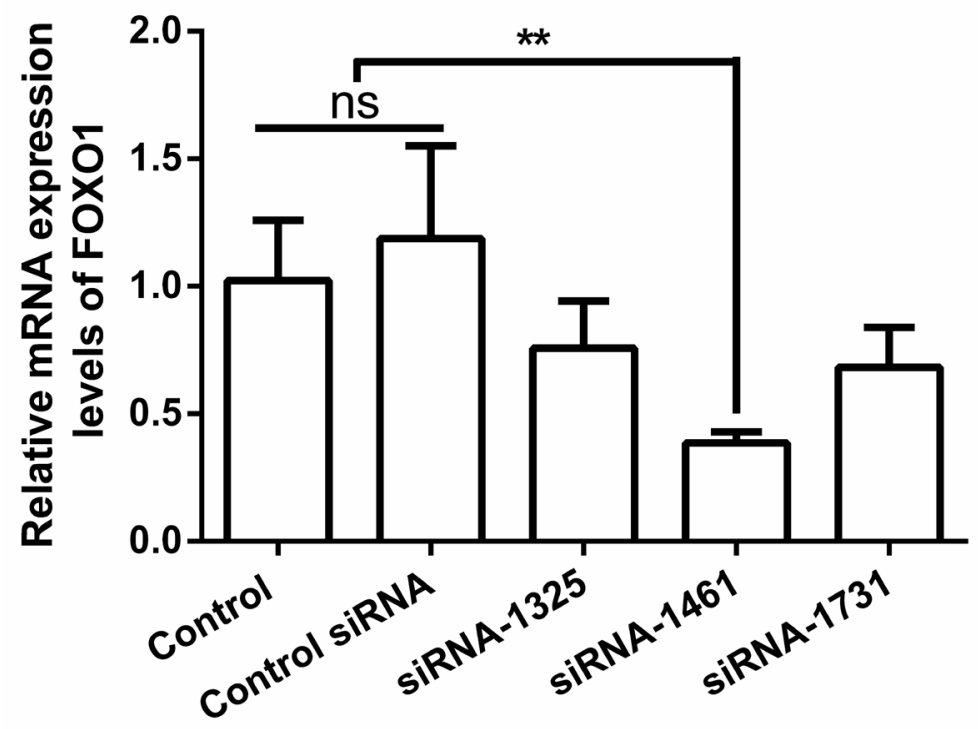

Figure 2. Silencing effect of different siRNA on forkhead box protein O1 (FOXO1) expression. Human C-33A cells were transfected with different siRNAs (siRNA-1325, siRNA-1461, or siRNA-1731) for $6 \mathrm{~h}$. FOXO1 gene expression was measured by quantitative polymerase chain reaction. Results showed that control siRNA did not affect the mRNA level of FOXO1. Transfection with siRNA-1461 significantly decreased the expression of FOXO1 as compared with siRNA-1325 or siRNA-1731 $(\mathrm{P}<0.05)$. Results are reported as means \pm SD of three separate experiments. $* * \mathrm{P}<0.05$. The experiment was performed three times with similar results.

Table 2. TNF- $\alpha$-induced apoptosis via FOXO1.

\begin{tabular}{lc}
\hline Group & Apoptosis (\%) \\
\hline Control & 17.4 \\
siRNA-1461 & 2.4 \\
siRNA-1461 + TNF- $\alpha$ & 3.0 \\
TNF- $\alpha$ & 34.0 \\
\hline
\end{tabular}

\section{Inhibition of FOXO1 represses TNF- $\alpha$-induced caspase activity}

Western blot analysis and qRT-PCR methods were used to assess the effect of FOXO1 on apoptosis involving TNF- $\alpha$-induced caspase activity. To study the effects of deficiency on the expression of FOXO1, this transcription factor was knocked down by using specific siRNA. As expected, siRNA-1461 significantly reduced the mRNA (Figure 4A) and protein (Figure 4B) expressions of the pro-apoptotic genes, caspase-3, 8, and $9(\mathrm{P}<0.05)$. However, TNF- $\alpha$ significantly increased the levels of these genes as compared with the control group (P $<0.05)$. In contrast, the control group showed no effects on TNF- $\alpha$-induced caspase activity. 

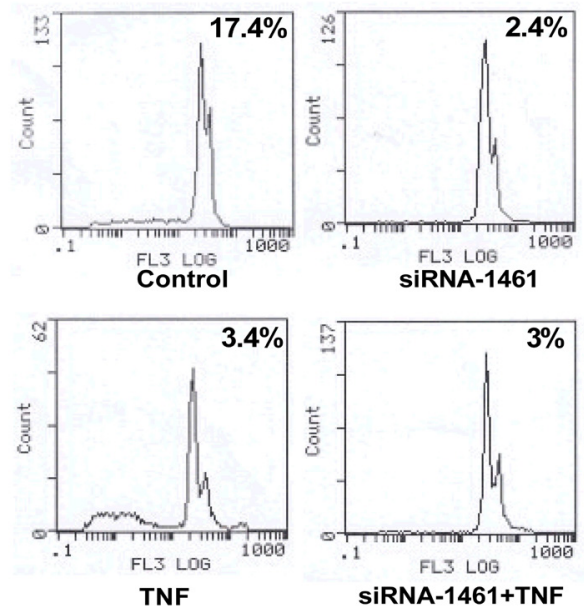

Figure 3. Forkhead box protein $\mathrm{O} 1$ (FOXO1) mediates tumor necrosis factor (TNF)- $\alpha$-induced apoptosis. C-33A cells were transfected with different siRNA for $6 \mathrm{~h}$ and then treated with $20 \mathrm{ng} / \mathrm{mL}$ TNF for $24 \mathrm{~h}$. The extent of apoptotic activity was tested by flow cytometry, which showed a significant difference among different groups. The results indicated that siRNA-1461 and siRNA-1461 + TNF- $\alpha$ groups showed significantly decrease apoptotic activity compared with control group $(\mathrm{P}<0.05)$. Apoptosis was significantly increased in TNF- $\alpha$-treated group as compared with control group $(\mathrm{P}<0.05)$. Data are reported as means $\pm \mathrm{SD}$ of three independent experiments $(* * \mathrm{P}<0.05)$.

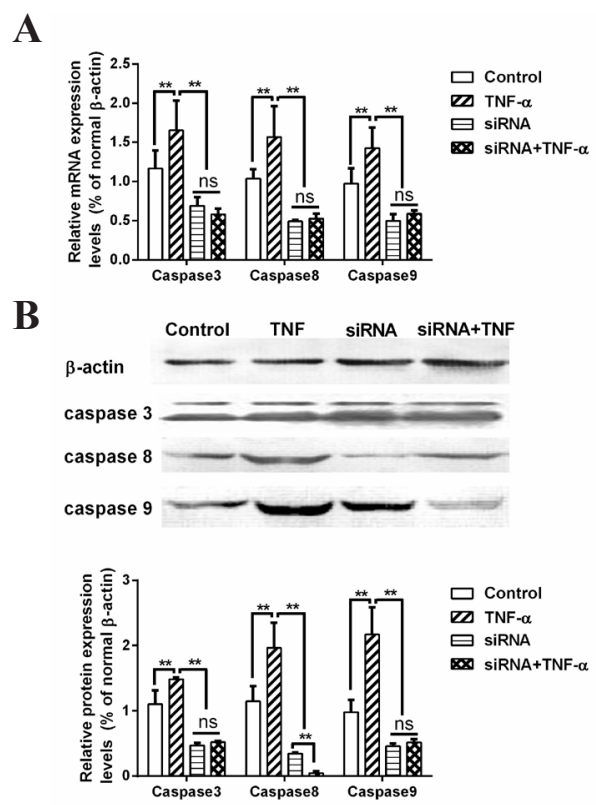

Figure 4. Silencing of forkhead box protein O1 (FOXO1) reduces tumor necrosis factor (TNF)- $\alpha$-induced caspase activity. Cells were transfected with siRNA-1469 for $6 \mathrm{~h}$ and stimulated with TNF- $\alpha(20 \mathrm{ng} / \mathrm{mL})$ for $24 \mathrm{~h}$. A. Real-time reverse transcriptase polymerase analysis of caspase- $3,-8$, and -9 expression. B. Western blot analysis of caspase-3, -8 , and -9 of the same samples. Beta actin staining was included as a loading control. Statistical analysis of caspase-3, -8 , and -9 expression after western blot quantification by using the Photoshop software. TNF- $\alpha$ significantly increased levels of caspase- $3,-8$, and -9 as compared with the control group $(\mathrm{P}<0.05)$. Results are reported as means $\pm \mathrm{SD}$ of three separate experiments $(* * \mathrm{P}<0.05)$. The experiment was performed three times with similar results. 


\section{DISCUSSION}

TNF- $\alpha$ is a pleiotropic cytokine, which performs various cellular activities, including apoptosis in several normal and disease-related processes. Previous reports demonstrate that TNF- $\alpha$ acts in multiple ways to enhance the expression of genes that promote apoptosis and diminish the expression of those that inhibit apoptosis (Alikhani et al., 2003).

Recent reports demonstrate that one of the crucial intracellular signaling events in TNF- $\alpha$-induced apoptosis is the sequential activation of caspases, a family of cysteine proteases (Chun et al., 2013). Two categories of caspases that are important for apoptosis have been recognized, including the initiators and executioner caspases. The initiator caspases such as caspase- 8 and -9 , are activated in the earlier phase of apoptosis, while the executioner caspases such as caspase-3, are activated by initiator caspases and are responsible for dismantling cells (Ravindran, 2009). Caspase-8 participates in the extrinsic (cytoplasmic) pathway of apoptosis whereas the intrinsic (mitochondrial) pathway is modulated by caspase-9 (Mcllwain et al., 2013). TNF- $\alpha$ induces apoptosis through activation of caspase- 3 by both caspase- 8 - and caspase-9-dependent pathways (Alikhani et al., 2004, 2005b).

Many studies have showed that the transcriptional regulator FOXO1 is involved in TNF- $\alpha$-induced apoptosis and mediates pro-apoptotic gene expression (Furukawa-Hibi et al., 2005; Alikhani et al., 2007) in fibroblasts, osteoblasts, and diabetes (Alikhani et al., 2005a; Alblowi et al., 2009; Behl et al., 2008). However, relatively little is known regarding its involvement in cervical cancer. We first examined the expression level of the transcriptional factor, FOXO1, in four different cervical cancer cell lines (HeLa, Caski, SiHa, and C-33A). Our results confirmed that two well-characterized cell lines, C-33A and Siha expressed FOXO1 at the highest and lowest levels, respectively (Zhang et al., 2015).

We therefore selected the C-33A cell line for further studies. We functionally silenced FOXO1 in the C-33A cell line by using a specific siRNA, and the central importance of FOXO1 was established through our experiments.

Our results suggest that silencing FOXO1 not only significantly reduced TNF$\alpha$-induced apoptosis but also decreased TNF- $\alpha$-induced caspase- $3,-8$, and -9 activities in cervical cancer cells. These results are similar to previous findings with other cancers (Alikhani et al., 2004, 2005b). Collectively, the presented data provide the first evidence that FOXO1 activation plays a vital role in TNF- $\alpha$-induced apoptosis. Furthermore, we provide the first evidence that FOXO1 is involved in mediating the extrinsic (cytoplasmic) and intrinsic (mitochondrial) apoptosis signaling pathways in cervical cancer cells. Taken together, we identified a novel apoptotic pathway involving a TNF- $\alpha$-FOXO1 transcriptional cascade, although the specific mechanism by which these actions are mediated remains to be clarified.

\section{ACKNOWLEDGMENTS}

We thank all of the research assistants and laboratory technicians who contributed to this study. We extend special thanks to Drs. Chang and Zhang for their helpful consultations during the preparation of the manuscript. Research supported by a grant from the Science and Technology Innovation Team Key Research Item of Jiamusi University (\#04099904). 


\section{REFERENCES}

Accili D and Arden KC (2004). FoxOs at the crossroads of cellular metabolism, differentiation, and transformation. Cell 117: 421-426.

Alblowi J, Kayal RA, Siqueira M, McKenzie E, et al. (2009). High levels of TNF- $\alpha$ contribute to accelerated loss of cartilage in diabetic fracture healing. Am. J. Pathol. 175: 1574-1584.

Alikhani M, Alikhani Z, He H, Liu R, et al. (2003). Lipopolysaccharides indirectly stimulate apoptosis and global induction of apoptotic genes in fibroblasts. J. Biol. Chem. 278: 52901-52908.

Alikhani M, Alikhani Z, Raptis M and Graves DT (2004). TNF- $\alpha$ in vivo stimulates apoptosis in fibroblasts through caspase-8 activation and modulates the expression of pro-apoptotic genes. J. Cell Physiol. 201: 341-348.

Alikhani M, Alikhani Z and Graves DT (2005a). FOXO1 functions as a master switch that regulates gene expression necessary for tumor necrosis factor-induced fibroblast apoptosis. J. Biol. Chem. 280: 12096-12102.

Alikhani M, Maclellan CM, Raptis M, Vora S, et al. (2007). Advanced glycation end products induce apoptosis infibroblasts through activation of ROS, MAP kinases, and the FOXO1 transcription factor. Am. J. Physiol. Cell Physiol. 292: C850-C856.

Alikhani Z, Alikhani M, Boyd CM, Nagao K, et al. (2005b). Advanced glycation end products enhance expression of proapoptotic genes and stimulate fibroblast apoptosis through cytoplasmic and mitochondrial pathways. J. Biol. Chem. 280: 12087-12095.

Behl Y, Siqueira M, Ortiz J, Li J, et al. (2008) Activation of the acquired immune response reduces coupled bone formation in response to a periodontal pathogen. J. Immunol. 181: 8711-8718.

Birkenkamp KU and Coffer PJ (2003). FOXO Transcription factors as regulators of immune homeostasis: molecules to die for? J. Immunol. 171: 1623-1629.

Brunet A, Bonni A, Zigmond MJ, Lin MZ, et al. (1999). Akt promotes cell survival by phosphorylating and inhibiting a Forkhead transcription factor. Cell 96: 857-868.

Burgering BM and Kops GJ (2002). Cell cycle and death control: long live Forkheads. Trends Biochem. Sci. 27:352-360.

Burgering BM and Medema RH (2003). Decision on life and death: FOXO Forkhead transcription factors are incomm and when PKB/Akt is off duty. J. Leukoc. Biol. 73: 689-701.

Calnan DR and Brunet A (2008). The FoxO code. Oncogene 27: 2276-2288.

Chun J, Joo EJ, Kang M and Kim YS (2013). Platycodin D induces anoikis and caspase-mediated apoptosis via p38 MAPK in AGS human gastric cancer cells. J. Cell Biochem. 114: 456-470.

Furukawa-Hibi Y, Kobayashi Y, Chen C and Motoyama N (2005). FOXO transcription factors in cell-cycle regulation and the response to oxidative stress. Antioxid. Redox Signal. 7: 752-760.

Gilley J, Coffer PJ and Ham J (2003). FOXO transcription factors directly activate bim gene expression and promote apoptosis in sympathetic neurons. J. Cell Biol. 162: 613-622.

Huang H and Tindall DJ (2007). Dynamic FoxO transcription factors. J. Cell Sci. 120: 2479-2487.

Mcllwain DR, Berger T and Mak TW (2013). Caspase functions in cell death and disease. Cold Spring Harb. Perspect. Biol. 5.

Navarro JF and Mora C (2005). Role of inflammation in diabetic complications. Nephrol. Dial. Transplant. 20: 2601-2604.

Navarro JF and Mora-Fernandez C (2006). The role of TNF-alpha in diabetic nephropathy: Pathogenic and therapeutic implications. Cytokine Growth Factor Rev. 17: 441-450.

Ravindran J, Prasad S and Aggarwal BB (2009). Curcumin and cancer cells: How many ways can curry kill tumor cells selectively? AAPS J. 11: 495-510.

Zhang B, Gui L, Zhao X, Zhu L and Li Q (2015). FOXO1 is a tumor suppressor in cervical cancer. Genet. Mol. Res. 14: 6605-6616. 